\title{
Kidney Transplant Modifies the Architecture and Microenvironment of Basal Cell Carcinomas
}

\author{
Anna Capasso ${ }^{a}$ Davide Viggiano $^{b}$ Michael W. Lee Le $^{a} f$ \\ Giuseppe Palladino $^{c}$ Giancarlo Bilancio $^{c}$ Mariadelina Simeoni ${ }^{b}$ \\ Giovanna Capolongo $^{\text {b }}$ Carmine Secondulfo $^{d}$ Andrea Ronchi $^{\mathrm{e}}$ \\ Alessandro Caputo $^{d}$ Pio Zeppa $^{d}$ Renato Franco ${ }^{\mathrm{e}}$ \\ a Department of Oncology, Livestrong Cancer Institutes, Dell Medical School, The University \\ of Texas at Austin, Health Learning Building, Austin, TX, USA; ${ }^{b}$ Department of Translational \\ Medical Sciences, University of Campania L. Vanvitelli, Naples, Italy; ${ }^{C}$ Nephrology Unit, \\ Salerno University Hospital, Salerno, Italy; ${ }^{d}$ Department of Medicine and Surgery, Schola

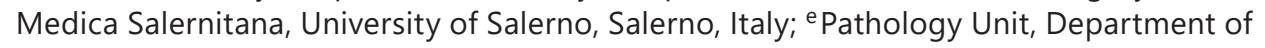 \\ Mental and Physical Health and Preventive Medicine, University of Campania L. Vanvitelli, \\ Naples, Italy; ${ }^{f}$ Department of Medical Education, Dell Medical School, University of Texas at \\ Austin, Austin, TX, USA
}

\section{Keywords}

Basal cells · Immunosuppression · Kidney transplantation · Onconephrology

\begin{abstract}
Background/Aims: Basal cell carcinoma (BCC) is a frequent type of nonmelanoma skin cancer, which shows a greater prevalence in kidney-transplanted (KT) patients than in the general population. The study of this tumor in KT patients may allow us to understand the influence of the tumor inflammatory microenvironment on cancer behavior, and to design new image analysis methods to determine prognosis and apply personalized medicine. The major hypothesis of the present work is that antirejection drugs, by modifying the B-cell/T-cell balance, induce measurable differences in tumoral cell microarchitecture and in the inflammatory microenvironment in KT patients compared to nontransplanted controls. Methods: In this retrospective study in an Italian cohort including $15 \mathrm{KT}$ patients and 15 control subjects from the general population who developed BCC, we analyzed tissue microarchitecture and inflammatory infiltrates of BCC using state-of-the-art nonlinear image analysis techniques such as fractal dimension and sample entropy of internuclear distances. Results: KT patients showed a nonsignificant trend to a greater number of nuclei in the basal cell layer compared to non-KT controls and subtle changes in the intact skin compared to controls. Similarly, the number of mitoses per unit length was almost doubled in the patients with KT compared to
\end{abstract}




\section{Kidney \\ Blood Pressure \\ Research}

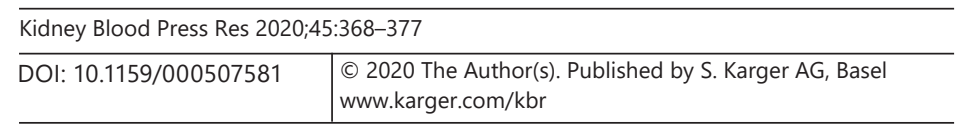

Capasso et al.: Kidney Transplants and Basal Cell Carcinomas

controls. However, when the number of mitotic cells was normalized by the total number of cells in the basal layer (mitotic index), these differences were not significant, although a clear trend was still present. Finally, KT patients showed a nonsignificant trend to an increased density of inflammatory cells close to the tumoral cell layer. When considering the intact skin, this difference was significant, with a $70 \%$ increase in the density of inflammatory cells. Conclusion: Data comparing the microarchitecture of BCC in normal subjects and KT patients are scanty, and the present study is the first to use nonlinear image analysis techniques to this aim. The observed differences underscore the relevance of T-cell suppression in cancer behavior. These data suggest that BCC develops in treated patients with specific biological characteristics which should be further analyzed in terms of therapeutic response.

(C) 2020 The Author(s)

Published by S. Karger AG, Basel

\section{Introduction}

Basal cell carcinoma (BCC) is one of the most common forms of skin cancer with an ageadjusted incidence of 366/100,000 for men and 293/100,000 for women [1]. Overall, the prognosis is good for BCC patients who receive treatment ( $>90 \%)$, and metastatic disease is the exception compared to melanomas or other forms of nonmelanoma skin cancers, including squamous cell carcinoma (SCC) [2-5]. The risk of developing BCC increases dramatically in the context of immunosuppression, such as in HIV patients or transplant recipients $[6,7]$. In kidney transplant (KT) recipients, the risk of BCC increases 10-16 times compared to healthy subjects [8-11]. This is probably due to the effect of long-term immunosuppression, which is needed to prevent graft rejection. Previous studies have characterized the clinical features of BCC in KT $[9,11-13]$.

Interestingly, immunosuppression in KT results in an up to 65-250 times higher risk of SCC $[8,14]$, so that the ratio of BCC:SCC favors SCC by 1:4 in KT, which is the opposite compared to the general population [15-18]. However, this may be a consequence of cancer registry indexing: in an Irish cohort with all skin cancers correctly registered, a 16-fold excess risk for BCC is present in KT patients compared to the age-matched general population [10]. The increased risk of tumors in KT subjects is one of the major topics in onconephrology [19].

However, the specific mechanism by which immunosuppression increases the risk of BCC remains to be determined. The relationship between cancer biology and the immune system has received increased attention in recent times, and this may help explain the association between BCC and KT [20]. Indeed, cancer is often accompanied by an inflammatory response, which is apparently not sufficient to stop tumor growth and metastasis [21]. The current use of drugs that stimulate T cells as anticancer agents (cancer immunotherapy) is clearly paralleled by the T-cell immunosuppressive activity of current antirejection therapies based on calcineurin inhibitors. The skin is apparently devoid of calcineurin (according to the Bio-GPS database and the Human Protein Atlas); therefore, the increased prevalence of BCC in KT is unlikely due to a direct effect of calcineurin inhibitors on the skin. Speculatively, the absence of $\mathrm{T}$ cells in the normal skin changes the biology of this tissue.

The presence of differences in tumoral cells and inflammatory responses in BCC between KT patients and nontransplanted controls is thus conceivable. Despite that, there are no studies available comparing the tissue architecture and the inflammatory microenvironment of BCC in KT patients compared to the general population. In this study, we have analyzed the tissue microarchitecture and inflammatory infiltrates of BCC in an Italian cohort including 15 KT patients and in 15 control subjects from the general population which developed BCC using state-of-the-art nonlinear image analysis techniques. 
Table 1. Clinical data of basal cell carcinomas in kidney-transplanted patients and in corresponding controls

\begin{tabular}{lll}
\hline & Kidney transplant $(n=15)$ & Controls $(n=15)$ \\
\hline Age, years & $59 \pm 2$ & $57 \pm 4$ \\
Gender & $\mathrm{F}=5, \mathrm{M}=10$ & $\mathrm{~F}=6, \mathrm{M}=9$ \\
Creatinine, $\mathrm{mg} / \mathrm{dL}$ & $1.2 \pm 0.4$ & $0.9 \pm 0.3$ \\
Immunosuppression, $n$ & Steroids: $11(73 \%)$ & None \\
& Cyclosporine: $8(53 \%)$ & \\
& Tacrolimus: $5(33 \%)$ & \\
& Mycophenolate: $11(73 \%)$ & \\
Antihypertensives drugs, $n$ & $13(86 \%)$ & $10(66 \%)$ \\
Comorbidities & Hypertension & Hypertension \\
\hline
\end{tabular}

\section{Patients and Methods}

\section{Patients}

This retrospective, observational study was performed in accordance with the ethics review board of the Ruggi Hospital and the University of Campania L. Vanvitelli. All transplanted patients underwent regular dermatological monitoring. Control subjects are members of the general population matched for age with KT patients. Exclusion criteria for control subjects were the presence of immunosuppressive states (apart from the use of antirejection drugs), genetic predisposition to tumors, and chronic inflammatory diseases. Exclusion criteria for KT patients were advanced chronic kidney disease (stages IV-V) and high corticosteroid use. All KT patients were treated with the calcineurin inhibitors cyclosporine $(n=$ $8)$ or tacrolimus $(n=7)$. Clinical data are reported in Table 1.

\section{Histological Processing}

Skin excisions were formalin-fixed, paraffin-embedded sections processed according to standardized procedures. They were stained with standard histological stains (H\&E, trichrome). Only H\&E staining was further considered in the present work. Diagnosis of BCC was performed by expert pathologists (A.C., A.R., P.Z., and R.F.).

\section{Image Analysis}

Whole sections were digitally acquired with a Ventana iScan HT slide scanner. Whole slide images were evaluated with the free software PMA.start by Pathomation (https://free. pathomation.com/), and single fields were then quantitatively analyzed using the ImageJ image analysis software (https://imagej.nih.gov/ij/).

For each subject, 3 fields of the intact skin and 3 of the basal layer of the tumor were assessed. Each field measured 1,112 ×696 $\mu \mathrm{m}$.

RGB images were first split in color channels (using the split channel tool), and only the monochromatic green channel was considered (because of the greatest signal from nuclei). The gray scale of the images was then inverted, so that the highest pixel values corresponded to greater nuclear staining intensity. Finally, the blue channel was subtracted from the average of green-red channels to improve the signal-noise ratio. The resulting images were then analyzed to identify the number of nuclei in the basal layer of both the tumor and the normal skin.

The following variables were quantified: number of nuclei per unit length, number of mitoses per total nuclei in the basal layer (percent of mitoses), and number of inflammatory cells per total nuclei in the basal layer (percent of inflammation). These data were measured by observers who were blinded to the patient group. 


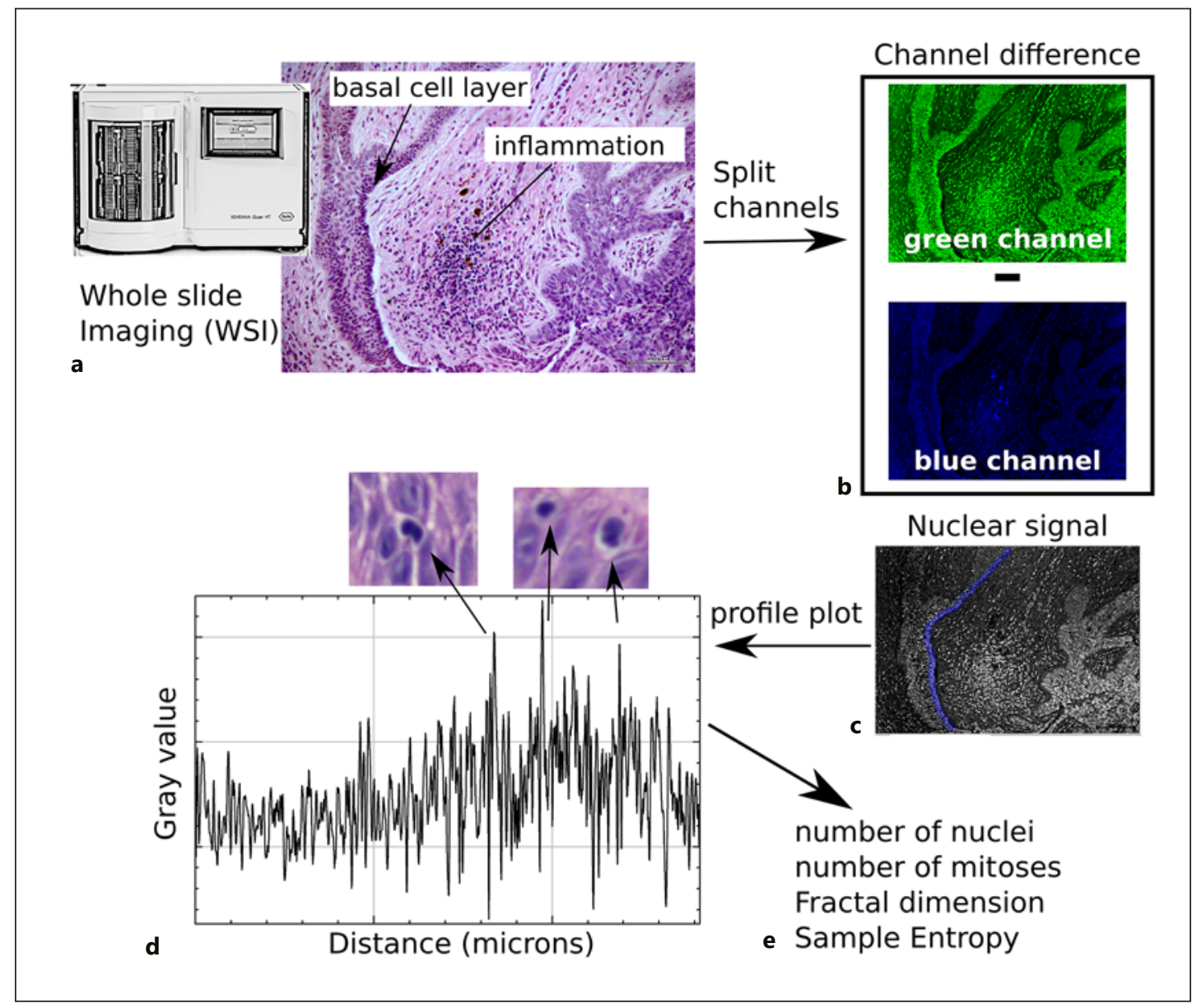

Fig. 1. Systematic pipeline of the image analysis system. BCC sections from control and KT patients have been scanned in order to analyze the entire section digitally at high resolution (a). The basal cell layer of the tumor and the inflammatory regions were then analyzed with ImageJ software. Images were separated into their color channels (b); the blue channel was then subtracted from the green channel to obtain only the nuclear signal (c). The nuclei were then manually selected with a polyline (evidenced in c) to obtain the gray values along the line (d). Each peak corresponds to a nucleus and the highest peaks to more intensely stained nuclei, possibly mitotic figures (insets). From the plot profile in $\mathbf{d}$, the number of nuclei per length, the number of mitoses, and the complexity of nuclear distribution (fractal dimension and entropy) are determined (e).

To obtain quantitative estimates of the nuclear distribution and number, a semiautomatic strategy was also pursued: a segmented line with a thickness adjusted to cover the height of the basal cell layer $(8 \mu \mathrm{m})$ was manually drawn onto the basal cell layer. The "plot profile" tool was then used to measure the average nuclear signal intensity along the line (intensity profile). An estimate of the number of nuclei per unit length was then obtained by counting the number of peaks on the intensity profile. Mitotic cells were identified by their greater signal intensity (which was due to the doubling of the DNA amount) by counting the peaks measuring 2 standard deviations above the average peak intensity. Nuclear distribution was then analyzed by measuring the entropy and fractal dimension of the intensity profile (Fig. 1). The variability in nuclear distribution was also assessed by measuring the standard deviation of internuclear distance over 50 nuclei (SDNN50). Automatic counting of cell nuclei was validated comparing the results with manual counts obtained by experienced operators in a pilot study based on 
Table 2. BCC architectural data and inflammatory features of the present series (see text for abbreviation)

\begin{tabular}{|c|c|c|c|}
\hline Variable & Controls & $\begin{array}{l}\text { Kidney } \\
\text { transplant }\end{array}$ & $\begin{array}{l}p \\
(t \text { test })\end{array}$ \\
\hline \multicolumn{4}{|l|}{ Tissue architecture } \\
\hline SDNN50 & $5.5 \pm 0.1$ & $5.3 \pm 0.3$ & 0.78 \\
\hline Sample entropy & $2.2 \pm 0.1$ & $2.0 \pm 0.3$ & 0.41 \\
\hline Fractal dimension & $1.19 \pm 0.02$ & $1.19 \pm 0.01$ & 0.76 \\
\hline \multicolumn{4}{|l|}{ Nuclear density, nuclei/ $\mu \mathrm{m}$} \\
\hline Total & $0.37 \pm 0.1$ & $0.44 \pm 0.3$ & 0.13 \\
\hline In intact skin & $0.35 \pm 0.02$ & $0.46 \pm 0.03$ & 0.01 \\
\hline \multicolumn{4}{|l|}{ Mitoses } \\
\hline Mitotic density, mitoses/mm & $0.96 \pm 0.3$ & $2.0 \pm 0.4$ & 0.03 \\
\hline Mitoses/total nuclei, \% & $0.29 \pm 0.08$ & $0.44 \pm 0.07$ & 0.2 \\
\hline \multicolumn{4}{|l|}{ Inflammatory cell density, cells/ $\mu \mathrm{m}$} \\
\hline Total & $0.34 \pm 0.04$ & $0.44 \pm 0.07$ & 0.19 \\
\hline In intact skin & $0.17 \pm 0.02$ & $0.27 \pm 0.07$ & 0.06 \\
\hline
\end{tabular}

20 different tumor images. Results showed excellent agreement between the automatic and the manual cell count (Pearson coefficient: $0.8, p<0.01$ ).

To measure the number of inflammatory cells, a $164-\mu \mathrm{m}$-thick ribbon was centered on the basal cell layer of the tumor using the "segmented line" tool and adjusting its thickness. The ribbon was then straightened using the "straighten" tool in ImageJ. Afterwards, a rectangular ROI from the end of the basal cell layer extending into the adjacent connective tissue by $67 \mu \mathrm{m}$ was selected, and the number of nuclei counted using a manually selected threshold and the "analyze particle" tool. Total inflammatory cells were then expressed as cells per unit length (referring to the length of the basal cell layer).

\section{Statistics}

Data were analyzed using the R environment. Differences between the KT and control group regarding the histological variables were tested using Student's $t$ test without the assumption of homogeneity of variance (Welsh method). Data are reported as means \pm SEM. Statistical significance was defined as $p<0.05$.

\section{Results}

The semiautomatic approach described in this study allows for objective measurement of the tissue architecture, the number of mitoses, and inflammatory microenvironment (Table 2).

The microarchitecture of the basal cell layer of KT patients was similar to that of controls: indeed, the spatial distribution of basal cell nuclei was similar in both groups when assessed by the entropy of the spatial distribution, the fractal dimension of nuclear distances, and SDNN50.

There was a nonsignificant trend to a greater number of nuclei in the basal cell layer in KT patients, which paralleled similar subtle changes in the intact skin compared to controls.

Similarly, the number of mitoses per unit length was almost doubled in the patients with KT compared to the controls. However, when the number of mitotic cells was normalized by the total number of cells in the basal layer (mitotic index), these differences were not significant, although a clear trend was still present. 


\section{Research}

Capasso et al.: Kidney Transplants and Basal Cell Carcinomas

Fig. 2. Major findings of the study concerning microarchitectural and inflammatory properties of BCC in transplanted patients. a Nuclear density is increased in the basal cell layer of BBC from treated KT patients. $\mathbf{b}$ The basal cell layer of BCC presents a greater number of mitoses in treated patients. c Trend to a greater number of inflammatory cells in the skin and BBC of treated patients compared to controls. Means \pm SEM. ${ }^{*} p<0.05$ vs. controls. c
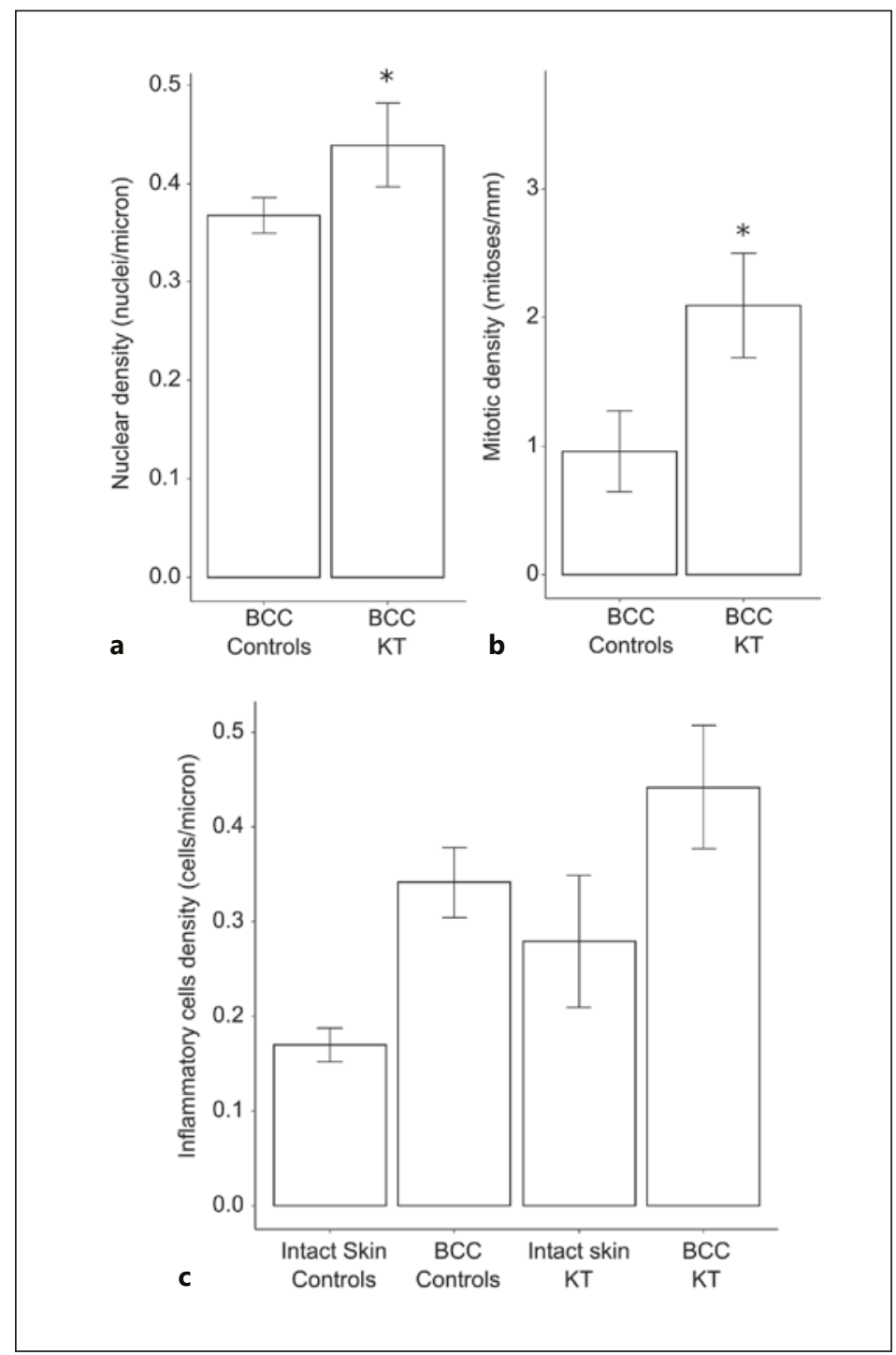

Finally, KT patients showed a nonsignificant trend to an increased density of inflammatory cells close to the tumor cell layer. Considering the intact skin, this difference was significant, with a $70 \%$ increase in the density of inflammatory cells. Figure 2 summarizes the main significant results. Figure 3 reports representative images describing differences in inflammatory components and nuclear density of BCC between controls and KT patients.

\section{Discussion}

The major finding of the present work is that the histoarchitecture and inflammatory microenvironment of BCC is different between KT patients and controls.

The molecular relationship between KT-associated immunosuppression and the development of BCC remains unclear and poorly defined. New diagnostic methods are needed to help discern the mediators involved in order that interventions can be designed to reduce the risk of developing BCC in these patients. To address this need, we created a 


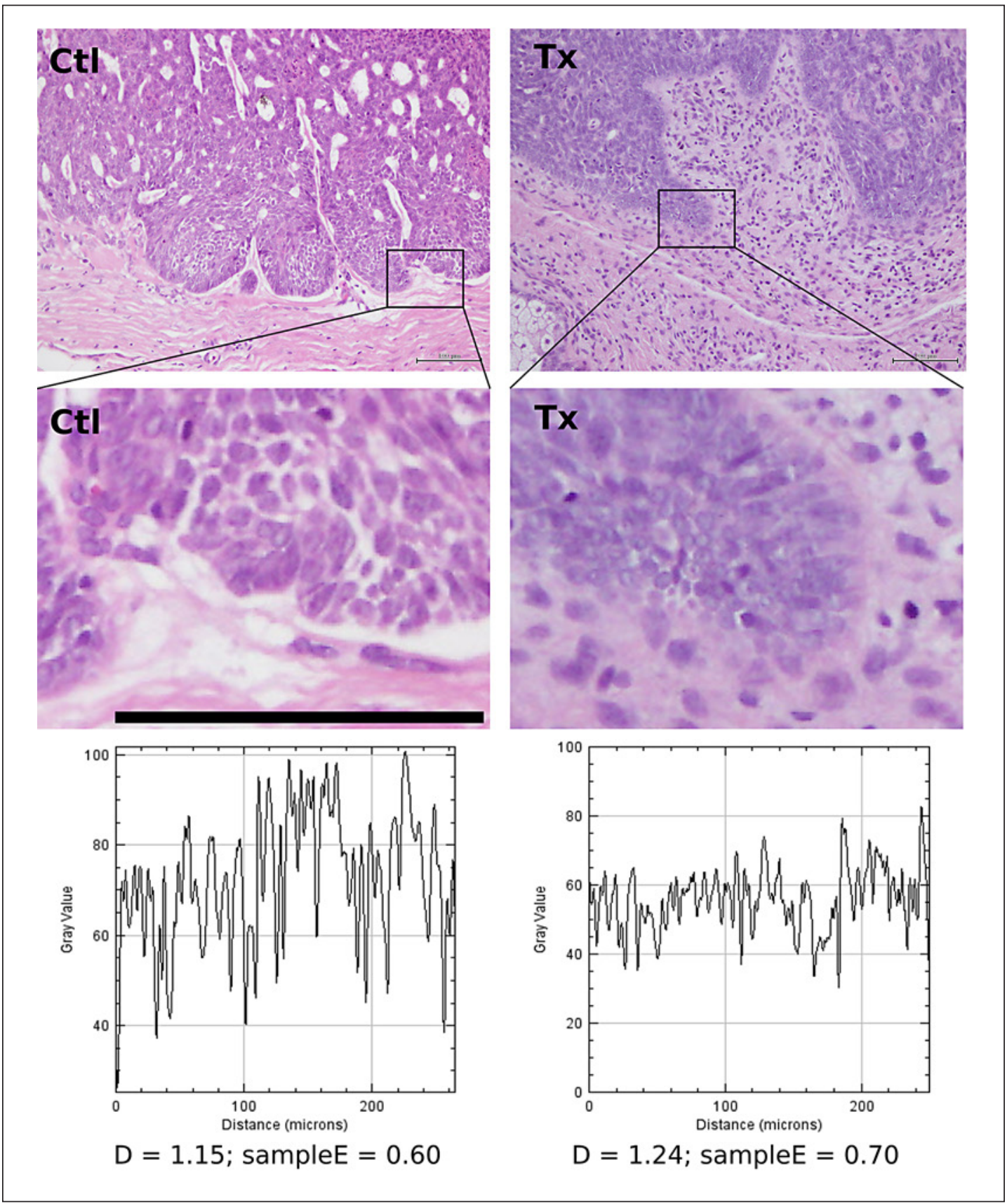

Fig. 3. Representative images of BCC in control (Ctl) and KT patients (Tx) at 2 magnification levels. Lower panels show the line plot of the signal intensity of the basal cell layer together with the resulting measurement of the fractal dimension (D) and sample entropy (sampleE).

pipeline image analysis strategy to evaluate and assess the histology of basal cell layers in KT patients with BCC. Previous observations showed that BCC was clinically similar in the KT and the general population [12]: BCC occurrence was increased at the level of the head and neck region at an advanced age and the level of the trunk at a younger age. Furthermore, the literature on gender prevalence is somewhat inconsistent regarding BCC in KT, but in the general population males are more often affected than females (as in our sample). Finally, BCC is reported to have poor or scanty inflammatory response in the general popu- 


\section{Kidney \\ Blood Pressure \\ Research}

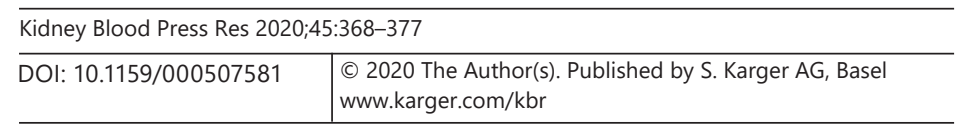

Capasso et al.: Kidney Transplants and Basal Cell Carcinomas

lation, although previous reports in KT patients [12] do not quantify the inflammatory response.

In this work, nonlinear analysis of histological data has been used to further quantify the tissue architecture. Specifically, we introduce SDNN50, sample entropy, and fractal dimension of nuclear distributions in the basal cell layer. The standard deviation of internuclear (NN) distances measures how much variability exists in the distance between nuclei. If all nuclei are all equally distanced ( $\mathrm{NN}=$ constant), the standard deviation of $\mathrm{NN}$ will be equal to zero. Conversely, any process that introduces irregular spacing would increase the variability in NN distances.

The sample entropy and the fractal dimension of NN sequences measure the complexity of these internuclear distances. These nonlinear measures reveal that a tissue with patches of closely packed nuclei alternate with widely spaced nuclei is substantially different from a tissue where the large spaces are randomly dispersed among closely spaced nuclei. Intuitively, in the first, the tissue is less complex than in the second one. Correspondingly, the sample entropy and the fractal dimension of the sequence of nuclear spacings are low when all nuclei are regularly spaced or grouped in homogeneous patches and increase up to a maximum when they are irregularly spaced. Entropy and fractal dimension have been already used to characterize histological images in other contexts [22-25].

Our findings reveal that BCC has unique features in KT patients compared to control subjects, including alterations in the tumor inflammatory microenvironment. Indeed, our data suggest that inflammatory infiltration in BCC is low in the general population but, importantly, also more frequent in KT patients. Given the relevance for immunotherapy of the tumor-infiltrating inflammatory cells, these data add useful information in the field [26].

This result is counterintuitive because a reduced inflammatory response is expected in the presence of immunosuppression. Indeed, skin inflammatory conditions occur in about $15 \%$ of KT patients versus $>60 \%$ in the general population [27]. In addition to inflammatory infiltrates, the tumor microarchitecture was also examined using image analysis. The fractal dimension, which is a measure of the complex nuclear distribution within the basal cell layer, and the sample entropy, a measurement of the information carried by the sequence of nuclear distances, were unchanged in both the KT patients and the general population. This suggests that the skin and tumor microarchitecture is preserved, and that only the number of nuclei and mitoses are affected. To our knowledge, no other reports on tumor structure and microenvironment in KT patients are published. Given the possibility that the phenomenon we describe in BCC could be present in other tumors as well, other studies are awaited in this exciting and new field of research.

This study has several limitations. The first pertains to the characterization of the inflammatory infiltrates. We could not obtain immunohistochemically stained tissues describing the type of inflammatory infiltrate (e.g., T vs. B cells). Using light microscopy, the infiltrates appear to be composed mainly of lymphocytes and few, if any, neutrophils. It is also plausible that these infiltrates are B cells in nature because the T cells should, in theory, be inhibited by immunosuppressive therapy [28]. Future studies should also consider the type of lymphocytic infiltrate by immunocytochemistry as this is very likely to be different in KT patients versus controls due to the anti-T-lymphocyte drugs in the former population.

A second limitation is the presence of several confounding variables between the KT and the control population. Although the immunosuppressive regimen is the most likely candidate to explain the different BCC behavior, it is not possible to exclude the possible contribution of comorbidities such as incipient diabetes, hypertension, polypharmacy, and reduced kidney function. A subgroup analysis of the KT patients did not reveal major effects of these factors; however, the small group size does not allow them to reach definitive conclusions. Nevertheless, given the paucity of data regarding this important subject, this first study is likely to 


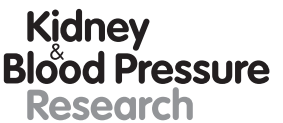

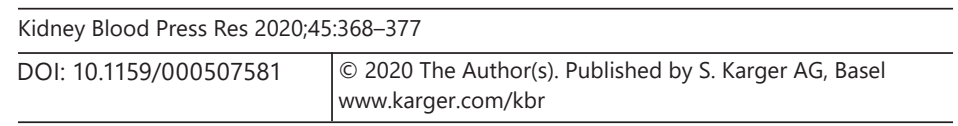

Capasso et al.: Kidney Transplants and Basal Cell Carcinomas

foster future observations with a larger sample size and better control of confounders. The small size of the population under study did not allow us to further classify the treated patients into subgroups. In the future, it would be interesting to examine potential differences between cyclosporine and tacrolimus treatments in a larger dataset.

Finally, our technique to measure the number of mitotic nuclei is based on the staining intensity of the nucleus; however, intraepithelial lymphocytes and (rarely) pyknotic cells may also show denser nuclei. This issue should be disentangled in future studies using markers of cell proliferation. However, a pilot study with manual counting of mitotic figures (not shown) gave overlapping results. The different inflammatory environment and microarchitecture of BCC in KT patients support a specific biology of these tumors in treated patients, possibly mediated by the altered immune system. Further clinical studies should, therefore, clarify whether temporary adjustments in immunosuppression may improve their characteristics.

\section{Statement of Ethics}

This retrospective study was approved by the Ethics Committee of the University of Campania L. Vanvitelli. The research complies with the guidelines for human studies and is ethically in accordance with the World Medical Association Declaration of Helsinki. Subjects provided their written informed consent.

\section{Disclosure Statement}

The authors have no conflicts of interest to disclose.

\section{Funding Sources}

This study was supported by the CPRIT Scholar Award \#RR160093.

\section{Author Contributions}

A.C., D.V., and M.W.L. provided the first draft of the manuscript. A.C., D.V., G.B., M.S., and G.C. provided the statistical analysis of the data. G.P., G.B., and C.S. recruited and analyzed patients; D.V., A.R., A.C., C.S., A.C., P.Z., and R.F. provided the image analysis. All authors modified the final draft of the manuscript.

\section{References}

1 Lloyd AR, Hales JP, Gandevia SC. Muscle strength, endurance and recovery in the post-infection fatigue syndrome. J Neurol Neurosurg Psychiatry. 1988 Oct;51(10):1316-22.

2 Hardie IR, Strong RW, Hartley LC, Woodruff PW, Clunie GJ. Skin cancer in Caucasian renal allograft recipients living in a subtropical climate. Surgery. 1980 Feb;87(2):177-83.

3 Rubin AI, Chen EH, Ratner D. Basal-cell carcinoma. N Engl J Med. 2005 Nov;353(21):2262-9.

4 de Rosa G, Vetrani A, Zeppa P, Zabatta A, Barra E, Gentile R, et al. Comparative morphometric analysis of aggressive and ordinary basal cell carcinoma of the skin. Cancer. 1990 Feb;65(3):544-9.

5 De Rosa G, Staibano S, Barra E, Zeppa P, Salvatore G, Vetrani A, et al. Nucleolar organizer regions in aggressive and nonaggressive basal cell carcinoma of the skin. Cancer. 1992 Jan;69(1):123-6.

6 Collins L, Quinn A, Stasko T. Skin Cancer and Immunosuppression. Dermatol Clin. 2019 Jan;37(1):83-94. 
7 España A, Redondo P, Fernández AL, Zabala M, Herreros J, Llorens R, et al. Skin cancer in heart transplant recipients. J Am Acad Dermatol. 1995 Mar;32(3):458-65.

8 Hartevelt MM, Bavinck JN, Kootte AM, Vermeer BJ, Vandenbroucke JP. Incidence of skin cancer after renal transplantation in The Netherlands. Transplantation. 1990 Mar;49(3):506-9.

9 Harwood CA, Proby CM, McGregor JM, Sheaff MT, Leigh IM, Cerio R. Clinicopathologic features of skin cancer in organ transplant recipients: a retrospective case-control series. J Am Acad Dermatol. 2006 Feb;54(2):290300.

10 Moloney FJ, Comber H, Conlon PJ, Murphy GM. The role of immunosuppression in the pathogenesis of basal cell carcinoma. Br J Dermatol. 2006 Apr;154(4):790-1.

11 Tran H, Chen K, Shumack S. Epidemiology and aetiology of basal cell carcinoma. Br J Dermatol. 2003 Nov; 149(s66 Suppl 66):50-2.

12 Mertz KD, Proske D, Kettelhack N, Kegel C, Keusch G, Schwarz A, et al. Basal cell carcinoma in a series of renal transplant recipients: epidemiology and clinicopathologic features. Int J Dermatol. 2010 Apr;49(4):385-9.

13 Kanitakis J, Alhaj-Ibrahim L, Euvrard S, Claudy A. Basal cell carcinomas developing in solid organ transplant recipients: clinicopathologic study of 176 cases. Arch Dermatol. 2003 Sep;139(9):1133-7.

14 Jensen P, Hansen S, Møller B, Leivestad T, Pfeffer P, Geiran O, et al. Skin cancer in kidney and heart transplant recipients and different long-term immunosuppressive therapy regimens. J Am Acad Dermatol. 1999 Feb; 40(2 Pt 1):177-86.

15 Euvrard S, Kanitakis J, Claudy A. Skin cancers after organ transplantation. N Engl J Med. 2003 Apr;348(17): 1681-91.

16 Bouwes Bavinck JN, Hardie DR, Green A, Cutmore S, MacNaught A, O'Sullivan B, et al. The risk of skin cancer in renal transplant recipients in Queensland, Australia. A follow-up study. Transplantation. 1996 Mar;61(5): 715-21.

17 Euvrard S, Kanitakis J, Pouteil-Noble C, Dureau G, Touraine JL, Faure M, et al. Comparative epidemiologic study of premalignant and malignant epithelial cutaneous lesions developing after kidney and heart transplantation. J Am Acad Dermatol. 1995 Aug;33(2 Pt 1):222-9.

18 Webb MC, Compton F, Andrews PA, Koffman CG. Skin tumours posttransplantation: a retrospective analysis of 28 years' experience at a single centre. Transplant Proc. 1997 Feb-Mar;29(1-2):828-30.

19 Capasso A, Benigni A, Capitanio U, Danesh FR, Di Marzo V, Gesualdo L, et al.; International Conference on OncoNephrology Participants. Summary of the International Conference on Onco-Nephrology: an emerging field in medicine. Kidney Int. 2019 Sep;96(3):555-67.

20 Wellenstein MD, de Visser KE. Cancer-Cell-Intrinsic Mechanisms Shaping the Tumor Immune Landscape. Immunity. 2018 Mar;48(3):399-416.

21 Gonzalez H, Hagerling C, Werb Z. Roles of the immune system in cancer: from tumor initiation to metastatic progression. Genes Dev. 2018 Oct;32(19-20):1267-84.

22 Viggiano D, Srivastava DP, Speranza L, Perrone-Capano C, Bellenchi GC, di Porzio U, et al. Quantifying barcodes of dendritic spines using entropy-based metrics. Sci Rep. 2015 Sep;5(1):14622.

23 Nigro M, Viggiano D, Ragone V, Trabace T, di Palma A, Rossini M, et al. A cross-sectional study on the relationship between hematological data and quantitative morphological indices from kidney biopsies in different glomerular diseases. BMC Nephrol. 2018 Mar;19(1):62.

24 Viggiano D, Speranza L, Crispino M, Bellenchi GC, di Porzio U, Iemolo A, et al. Information content of dendritic spines after motor learning. Behav Brain Res. 2018 Jan;336:256-60.

25 Ji MY, Yuan L, Jiang XD, Zeng Z, Zhan N, Huang PX, et al. Nuclear shape, architecture and orientation features from H\&E images are able to predict recurrence in node-negative gastric adenocarcinoma. J Transl Med. 2019 Mar; 17(1):92.

26 Steinel NC, Lee EM, Viggiano D, Capasso A, Lee MW. The renal adverse effects of cancer immunotherapy. J Nephrol. 2020 Jan,DOI: 10.1007/s40620-019-00691-2.

27 Savoia P, Cavaliere G, Zavattaro E, Veronese F, Fava P. Inflammatory Cutaneous Diseases in Renal Transplant Recipients. Int J Mol Sci. 2016 Aug;17(8):E1362.

28 Crespo E, Fernandez L, Lúcia M, Melilli E, Lauzurica R, Penin RM, et al. Effector Antitumor and Regulatory T Cell Responses Influence the Development of Nonmelanoma Skin Cancer in Kidney Transplant Patients. Transplantation. 2017 Sep;101(9):2102-10. 